\title{
Review Article \\ The Role of Uric Acid in Kidney Fibrosis: Experimental Evidences for the Causal Relationship
}

\author{
Il Young Kim, ${ }^{1,2}$ Dong Won Lee, ${ }^{1,2}$ Soo Bong Lee, ${ }^{1,2}$ and Ihm Soo Kwak ${ }^{1,3}$ \\ ${ }^{1}$ Division of Nephrology, Department of Internal Medicine, Pusan National University School of Medicine, \\ Yangsan 626-770, Republic of Korea \\ ${ }^{2}$ Research Institute for Convergence of Biomedical Science and Technology, Pusan National University Yangsan Hospital, \\ Yangsan 626-770, Republic of Korea \\ ${ }^{3}$ Medical Research Institute, Pusan National University Hospital, Busan 602-739, Republic of Korea \\ Correspondence should be addressed to Soo Bong Lee; sbleemd@pusan.ac.kr
}

Received 27 February 2014; Revised 5 April 2014; Accepted 21 April 2014; Published 5 May 2014

Academic Editor: Keizo Kanasaki

Copyright (C) 2014 Il Young Kim et al. This is an open access article distributed under the Creative Commons Attribution License, which permits unrestricted use, distribution, and reproduction in any medium, provided the original work is properly cited.

Hyperuricemia is a common finding in chronic kidney disease due to decreased uric acid clearance. The role of uric acid as a risk factor for chronic kidney disease has been largely debated, and recent studies suggested a role of uric acid in the causation and progression of kidney fibrosis, a final common pathway in chronic kidney disease. Uric acid and xanthine oxidase may contribute to kidney fibrosis mainly by inducing inflammation, endothelial dysfunction, oxidative stress, and activation of the reninangiotensin system. Besides, hyperuricemia induces alterations in renal hemodynamics via afferent arteriolopathy and contributes to the onset and progression of kidney fibrosis. Xanthine oxidase inhibitors may prevent kidney damage via lowering uric acid and/or inhibiting xanthine oxidase. However, there is still no sufficient evidence from interventional clinical researches supporting the causal relationship between uric acid and kidney fibrosis. The effect and role of xanthine oxidase inhibitors in preventing kidney fibrosis and chronic kidney disease progression must be further explored by performing future large scale clinical trials.

\section{Introduction}

Regardless of the underlying etiology, most forms of chronic kidney disease (CKD) are characterized by progressive fibrosis as a final common pathway, which eventually affects all substructures of the kidney leading to a final consequence of end-stage renal disease. Although there has been a great deal of research, a comprehensive understanding of the pathogenetic mechanisms of kidney fibrosis remains uncertain and this hampers the development of effective therapeutic strategies [1].

Uric acid (UA) is the final breakdown product of purine degradation in humans, and elevated serum UA level, hyperuricemia, is causative in gout and urolithiasis due to the formation and deposition of monosodium urate crystals. Hyperuricemia is a common finding in CKD due to decreased UA clearance. Its role as a risk factor for CKD progression has been largely debated, and it was primarily considered as a marker or epiphenomenon of kidney damage $[2,3]$. However, during the last 2 decades, accumulating evidences have suggested a role of UA in the causation or progression of cardiovascular diseases and CKD [3-9]. Therefore, UA lowering therapy with xanthine oxidase (XO) inhibitors, which are already being widely used in the treatment of gout, could be promising for preventing the progression of CKD even in patients without hyperuricemia; however, solid clinical evidence is still lacking. To promote large scale prospective clinical trials, it is essential to accumulate experimental evidences for the cause-effect relationship between UA and kidney fibrosis.

In this review, after providing a brief background concerning UA physiopathology, we will focus on the mechanistic role of UA in kidney fibrosis. We will also review the role of $\mathrm{XO}$ and the effect of $\mathrm{XO}$ inhibitors in preventing kidney fibrosis and their associated mechanisms. 


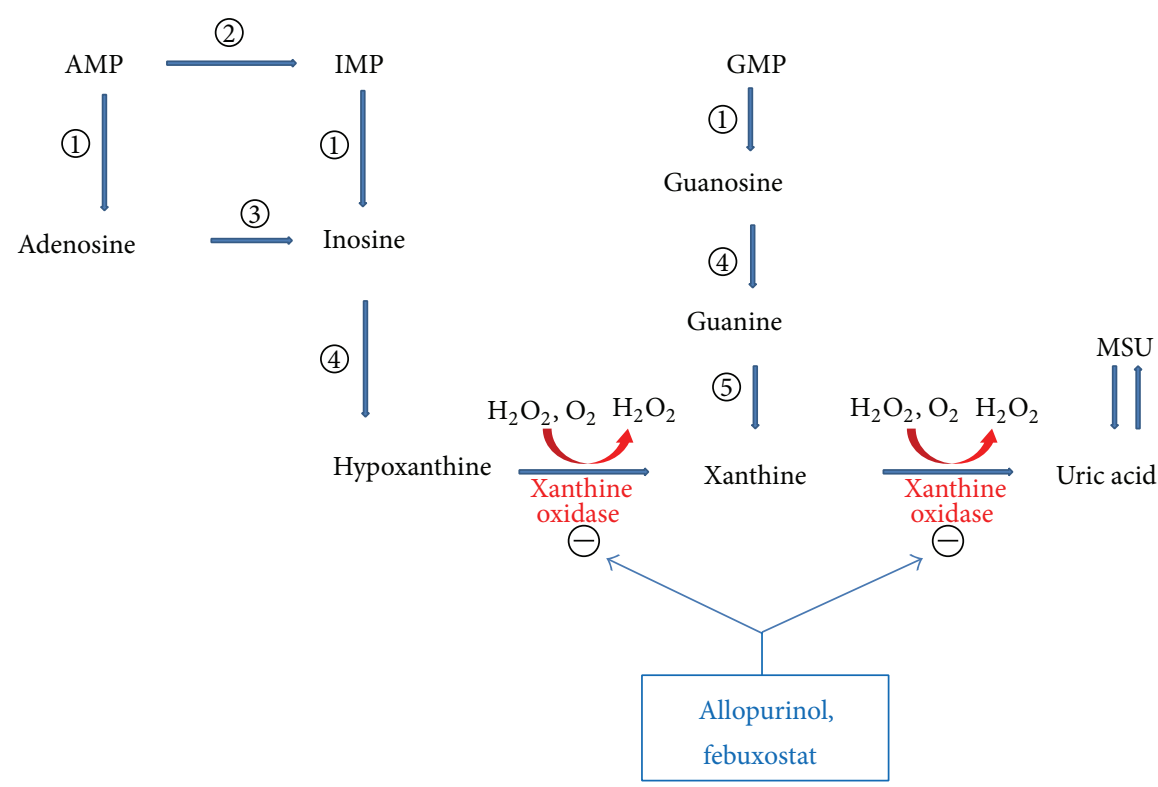

FIgURE 1: The pathway of purine nucleotides degradation in humans showing the competitive inhibition of uric acid formation by xanthine oxidase inhibitors and the site of action. AMP: adenosine monophosphate; GMP: guanosine monophosphate; IMP: inosine monophosphate; MSU: monosodium urate; (1): $5^{\prime}$-nucleotidase; (2): AMP deaminase; (3): adenosine deaminase; (4): purine nucleoside phosphorylase; (5): guanine deaminase.

\section{Physiopathology of Uric Acid}

Cell turnover leads to the production of adenosine, inosine, and guanosine. They degrade to hypoxanthine and xanthine, which are the substrates for the widely distributed $\mathrm{XO}$ in the formation of UA. XO catalyzes the oxidation of purine substrates, xanthine and hypoxanthine, producing both UA and reactive oxygen species (ROS). Thus, $\mathrm{XO}$ is one of the major enzymatic sources of ROS. Allopurinol and febuxostat are inhibitors of $\mathrm{XO}$, and they reduce uric acid and ROS formation (Figure 1) [10].

UA is the oxidation end-product of purine metabolism in humans and higher primates. Most other mammals, except for the Dalmatian dogs, can degrade UA further to watersoluble allantoin with the enzyme uricase, and as a result serum urate levels are about $10 \%$ of those in humans $[9,11]$. However, in humans and higher primates, mutations in the uricase gene occurred during evolution and, making the enzyme nonfunctional, resulted in higher levels of serum UA than in other mammals [12].

Urates are the ionized form of UA, and, at a physiologic $\mathrm{pH}$ of 7.4 , over $95 \%$ of UA dissociates into urates, with $98 \%$ existing as monosodium urate. The serum urate level depends on dietary purines, the breakdown of endogenous purines, and the renal and intestinal excretion of urate. Hyperuricemia is defined as the accumulation of serum UA beyond its solubility point in water $(6.8 \mathrm{mg} / \mathrm{dL})$, and it develops due to UA overproduction, undersecretion, or both [13]. The dominating factor contributing to hyperuricemia is underexcretion of urate [11]. Under normal conditions, $70 \%$ of the UA produced is eliminated in the urine and the remaining UA is removed via biliary secretion.
In the kidney, urate is easily filtered through the glomerulus and subsequently reabsorbed by the proximal tubule cells of the kidney and after further absorption, about $10 \%$ of urate is finally excreted [14]. An anion exchanger and a voltagedependent pathway seem to be the mechanisms involved in urate transport [15].

Allopurinol, a purine inhibitor of $\mathrm{XO}$, has been conventionally used for urate-lowering therapy to inhibit UA synthesis. A novel urate-lowering drug, febuxostat, is a potent nonpurine selective inhibitor of $\mathrm{XO}$, and it inhibits both the reduced and oxidized forms of the enzyme in contrast to allopurinol that inhibits the reduced form of the enzyme only $[16,17]$. Febuxostat is metabolized mainly by glucuronidation and oxidation in the liver, has its dual (urinary and fecal) pathways in excretion (urinary and fecal excretion rates: $49.1 \%$ and $44.9 \%$, resp.), and is effective and well tolerated in patients with mild to moderate renal and hepatic impairment $[18,19]$. Animal studies have demonstrated that febuxostat has a greater UA-lowering effect than allopurinol [20, 21]. Febuxostat's chemical structure does not resemble a pyrimidine or purine and is unlike that of allopurinol [22]. It does not inhibit other enzymes involved in purine or pyrimidine metabolism [23].

Studying the role of UA in kidney fibrosis, a process which eventually leads to $\mathrm{CKD}$, is very difficult since uric acid is excreted primarily by the kidney, and hence a decrease in the glomerular filtration rate (GFR) is inevitably accompanied by a rise in the serum UA level. As such, studies in experimental animals in which serum UA can be modulated are critical to understand whether there is a role for UA in the causation or progression of CKD [7]. 


\section{Experimental Studies Supporting the Roles of Uric Acid and Xanthine Oxidase in Kidney Fibrosis}

In the past, hyperuricemia was thought to cause CKD, the so-called urate nephropathy, by the deposition of urate crystals in the renal interstitium. This results in a chronic inflammatory response and in progressive tubulointerstitial injury in a similar manner as seen with tophi in gouty arthritis [24]. However, the pathologic role of hyperuricemia in kidney disease by a crystal-independent mechanism is somewhat less clear.

3.1. Hyperuricemic Rat Models and Types of Kidney Injury. Generating hyperuricemia in laboratory animals proved to be difficult due to the fact that most mammals have the uricase enzyme. Rodent models in which the uricase gene was knocked out showed renal failure due to extensive tubular crystal deposition and finally death [25]. An alternative model with milder degree of hyperuricemia without crystal deposition, which is more applicable to human disease, was developed using uricase inhibitor, oxonic acid (OA) [26-29]. It is also possible to lower serum UA levels using the $\mathrm{XO}$ inhibitors such as allopurinol and febuxostat.

After the year 2001, when the hyperuricemic rat model was developed by using OA [26] and until recently, there have been accumulating experimental evidences that hyperuricemia induced renal injury, which may be prevented by lowering serum UA levels with XO inhibitors. The first study using OA-induced hyperuricemic rat model demonstrated that hyperuricemia induced systemic hypertension as well as ischemic type of kidney injury with collagen deposition, macrophage infiltration, and increase in tubular expression of osteopontin documented via immunohistochemical stains. The kidneys were devoid of urate crystals and were normal by light microscopy. Blood pressure was lowered by reducing serum UA levels with allopurinol. Hyperuricemic rats treated with OA also showed an increase in juxtaglomerular renin and a decrease in macula densa neuronal nitric oxide (NO) synthase. Both the kidney injury and hypertension were attenuated by treatment with renin-angiotensin system (RAS) blocker (enalapril) or a substrate for endothelial NO synthase (L-arginine). This study suggested that UA induced hypertension and renal injury via a crystal-independent mechanism with the activation of RAS and inhibition of NO synthase [26]. Another study demonstrated that hyperuricemia induced arteriolopathy of the afferent arteriole by a blood pressure-independent mechanism. In this study, hyperuricemic rats fed OA showed hypertension and afferent arteriolar thickening. Allopurinol prevented hyperuricemia, hypertension, and arteriolopathy. Controlling blood pressure with hydrochlorothiazide did not prevent hyperuricemia and arteriolopathy, suggesting that hyperuricemia-induced arteriolopathy was not mediated by blood pressure. This study also showed that arteriolopathy was mediated by the direct effect of UA on proliferation of vascular smooth muscle cells with activation of RAS [27].
In another rat model with dietary intake of adenine, which may be the source of the UA as a purine base, adeninefed rats showed hyperuricemia [30, 31]. Adenine-fed rats also showed increased kidney inflammation (TNF- $\alpha$ ), fibrotic (TGF- $\beta$ ), and oxidative (HO-1) markers, along with pathologically confirmed kidney fibrosis. Lowering of UA levels with allopurinol reversed the kidney damage, suggesting that UA played a major role in the pathogenesis of kidney fibrosis [30]. Another animal model of tubulointerstitial nephritis (TIN) induced by excessive adenine intake exhibited significant renal dysfunction and enhanced cellular infiltration accompanied by collagen deposition. It also showed higher gene and protein expression of proinflammatory cytokines. Treatment with allopurinol led to reduced levels of uric acid, oxidative stress, and collagen deposition and a downregulation of the nuclear factor-kB (NF-kB) signaling pathway [31].

Based on the growing evidence that lowering UA levels with allopurinol prevented renal injury induced by hyperuricemia, the role of febuxostat in preventing kidney injury was investigated. In OA-induced hyperuricemic rats, febuxostat lowered UA levels and ameliorated systemic and glomerular hypertension as well as preglomerular arteriolopathy. In normal rats without hyperuricemia, febuxostat tended to lower UA levels and had no effect on blood pressure, glomerular pressure, and afferent arteriole morphology [32].

\subsection{Systemic and Glomerular Hypertension in Hyperuricemia.} There are some experimental studies that demonstrated the relationship between UA and renal hemodynamics. In one study, hyperuricemic rats fed OA not only developed systemic hypertension but also glomerular hypertension. Hyperuricemic rats showed increased glomerular capillary pressure with afferent arteriole thickening. Allopurinol prevented hyperuricemia, systemic and glomerular hypertension, and arteriolopathy. Glomerular capillary pressure and arteriolar thickening correlated with serum UA and systolic blood pressure. This study suggested that glomerular hypertension might be mediated by insufficient vasoconstriction of the afferent arteriole to systemic hypertension, allowing the transmission of systemic pressure to the glomerular capillary tuft [33]. In another study, hyperuricemia induced by OA resulted in renal cortical vasoconstriction and glomerular hypertension due to afferent arteriole thickening in normal and remnant kidney rats. Allopurinol prevented structural and functional alterations in both normal and remnant kidney rats. This study suggested that hyperuricemia-induced glomerular alterations caused renal ischemia, which in turn induced tubulointerstitial inflammation and fibrosis [28].

\subsection{Renal Progression in Animal Models of Chronic Kidney} Disease. Hyperuricemia also contributed to renal progression in animal models of CKD. In 5/6 nephrectomy remnant kidney model, remnant kidney rats fed OA showed more severe renal failure, proteinuria, and histologic findings (thickening of the preglomerular arteries, glomerulosclerosis, and interstitial fibrosis) compared to remnant kidney rats without hyperuricemia. Allopurinol reduced serum UA levels 
and prevented the functional and histologic changes in remnant kidney rats fed OA. This study also demonstrated that hyperuricemia accelerated renal progression by increasing renin expression in the renal cortex and cyclooxygenase2 (COX-2) expression in the afferent arteriole. In particular, this study showed that increased COX-2 expression induced by hyperuricemia was associated with proliferation of vascular smooth muscle cells in preglomerular arteries [29]. In another 5/6 nephrectomy rat model, remnant kidney rats treated with OA developed hyperuricemia, renal vasoconstriction, and glomerular hypertension in association with further aggravation of afferent arteriolopathy compared to remnant kidney rats that were not treated with OA. Febuxostat prevented hyperuricemia and ameliorated renal injury in remnant kidney rats treated with OA. Interestingly, febuxostat had a comparable beneficial effect in both remnant kidney rats with hyperuricemia (treated with OA) and remnant kidney rats without hyperuricemia (not treated with OA) [34].

3.4. Exacerbation of Renal Injury in Animal Models of Cyclosporine and Diabetic Nephropathy. Hyperuricemia has also been known to exacerbate renal injury in some animal disease models including cyclosporine (CsA) and diabetic nephropathy. Hyperuricemia frequently complicated CsA therapy. In one study using a model of CsA nephropathy, the rats developed hyperuricemia with arteriolar hyalinosis, tubular injury, and interstitial fibrosis. CsA nephropathy rats fed OA showed higher UA levels with more severe histologic findings compared to CsA nephropathy rats that were not treated with OA. This study also demonstrated that the mechanism did not involve intrarenal urate crystal deposition and appeared to involve activation of RAS and inhibition of intrarenal NO production [35]. In another study, CsA-treated rats developed hyperuricemia with arteriolar hyalinosis, tubular atrophy, interstitial fibrosis, increased cell proliferation, and decreased vascular endothelial growth factor (VEGF). Treatment with allopurinol or a uricosuric, benzbromarone, reduced the severity of the kidney injury. Both drugs provided comparable protection and the similar protection observed with both drugs suggests that the effect is associated more with lowering UA levels than the antioxidant effect of allopurinol [36]. Hyperuricemia has recently been recognized to be a risk factor for nephropathy in the diabetic subject. Diabetic $(\mathrm{db} / \mathrm{db})$ mice developed hyperuricemia, albuminuria, mesangial matrix expansion, and mild tubulointerstitial disease. Allopurinol treatment not only reduced UA levels but also reduced albuminuria and ameliorated tubulointerstitial injury. The mechanism for protection was shown to be due to a reduction in inflammatory cells mediated by a reduction in ICAM-1 expression by tubular epithelial cells [37]. In another diabetic nephropathy model using KK-A(y)Ta mice, lowering UA levels with allopurinol attenuated transforming growth factor- (TGF-) $\beta 1$-induced profibrogenic progression in the mice, suggesting that lowering serum UA may be an effective therapeutic intervention to prevent the progression of diabetic nephropathy [38].
3.5. Oxidative Stress and Endothelial Dysfunction in Hyperuricemia. While UA has been reported to be a potent antioxidant in the extracellular fluid [39], it has prooxidative effect once inside the cell $[40,41]$. According to a hypothesis [39], the silencing of the uricase gene with an increase in the blood level of UA provided an evolutionary advantage for ancestors of Homo sapiens. This hypothesis was based on in vitro experiments which showed that UA is a powerful scavenger of singlet oxygen, peroxyl radicals, and hydroxyl radicals. UA circulating at an elevated level was proposed to be one of the major antioxidants of the plasma that protects cells from oxidative damage, thereby contributing to an increase in life span of human species and decreasing the risk of cancer [42]. On the other hand, a vast literature on the epidemiology of cardiovascular disease, hypertension, and metabolic syndrome overwhelmingly shows that, at least among modern Homo sapiens, a high level of UA is strongly associated with and in many cases predicts development of hypertension, visceral obesity, insulin resistance, dyslipidemia, diabetes, kidney disease, and cardiovascular and cerebrovascular events [42]. Antioxidant effect of UA varies according to the presence of specific components, in different physiochemical circumstances and in various compartments of the human body. UA is an antioxidant only in the hydrophilic environment and even in the plasma UA can prevent lipid peroxidation only as long as ascorbic acid is present [43]. Major sites where the antioxidant effects have been proposed are the central nervous system [44, 45], liver [46], and heart [47].

In OA-induced hyperuricemic rat model, hyperuricemia caused intrarenal oxidative stress, increased expression of NOX-4 subunit of renal NADPH oxidase and angiotensin II, and decreased NO bioavailability. Hyperuricemic rats also showed systemic hypertension, renal vasoconstriction, and arteriolopathy. Tempol (superoxide scavenger) attenuated the adverse effect induced by hyperuricemia despite equivalent hyperuricemia, suggesting that UA might cause oxidative stress [48]. In another study, hyperuricemic rats treated with $\mathrm{OA}$ showed decreased urinary $\mathrm{NO}$ metabolites $\left(\mathrm{NO}_{2}{ }^{-} / \mathrm{NO}_{3}{ }^{-}\right)$, systemic hypertension, renal vasoconstriction, and preglomerular arteriolopathy. Chronic administration of L-arginine, a substrate for endothelial NO synthase, increased the urinary excretion of $\mathrm{NO}_{2}{ }^{-} / \mathrm{NO}_{3}{ }^{-}$and preserved arteriolar structures probably mediated by the antiproliferative effect of $\mathrm{NO}$ on vascular smooth muscle cells, suggesting the role of endothelial dysfunction as a mediator of renal injury induced by hyperuricemia [49]. A recent study reported that UA-induced endothelial dysfunction was associated with mitochondrial alterations and decreased intracellular ATP concentrations [50]. An experimental model of streptozotocin-induced diabetic rats showed that febuxostat improved endothelial dysfunction via attenuating oxidative stress by XO inhibition [51].

3.6. Hyperuricemia and Inflammatory Responses. UA induces inflammatory responses. Hyperuricemia-induced inflammatory response mediates kidney injury via alteration of vascular and tubular cells in kidney. UA has the ability to induce 
monocyte chemoattractant protein- (MCP-) 1 in vascular smooth muscle cells, suggesting that it may have a role in the vascular changes associated with hypertension and vascular disease [52]. UA also contributes to kidney damage through vascular cell proliferation induced by activation of COX-2 [29] and increased expression of C-reactive protein (CRP) [53]. UA has been known to inhibit renal proximal tubule cell proliferation via activation of NF- $\kappa \mathrm{B}$ and cytoplasmic phospholipase $\mathrm{A}_{2}$ [54]. Hyperuricemia also increases extracellular matrix (ECM) synthesis through upregulation of lysyl oxidase (LOX) expression in renal tubular epithelial cells [55]. UA contributes to tubulointerstitial inflammation by inducing expression of intracellular adhesion molecule(ICAM-) 1 in renal tubular epithelial cells [37].

$\mathrm{XO}$ has been reported to be upregulated by various inflammatory stimuli such as lipopolysaccharide (LPS), hypoxia, and cytokines [56-60]. Augmented XO eventually causes excess ROS formation, leading to tissue damage. Pharmacological inhibitors of XO, such as allopurinol and febuxostat, have been reported to have an anti-inflammatory effect in various diseases such as atherosclerosis, congestive heart failure, acute lung injury, renal interstitial fibrosis, and ischemia-reperfusion injury [61-66]. A recent study suggested a molecular mechanism underlying the involvement of $\mathrm{XO}$ in inflammatory pathways, and it also suggested that XO mediates LPS-induced phosphorylation of JNK through ROS production and MKP-1 inactivation, leading to MCP-1 production in macrophages. Febuxostat significantly suppressed LPS-induced MCP-1 production in human macrophages and in vivo in mice [56].

\subsection{Hyperuricemia and Epithelial-Mesenchymal Transition.} In the last decade, epithelial-mesenchymal transition (EMT), a process by which fully differentiated epithelial cells lose their epithelial characteristics and undergo phenotypic conversion to mesenchymal cells, has emerged as an important pathway leading to generation of matrix-producing fibroblasts and myofibroblasts in kidney fibrosis. In addition to kidney fibrosis, EMT has been known to play a pivotal role in embryonic development, wound healing, tissue regeneration, and cancer progression $[67,68]$.

A recent study showed that UA exerted a direct effect on renal tubular cells by inducing EMT [69]. OA-induced hyperuricemic rats showed evidence of EMT before the development of significant tubulointerstitial fibrosis at 4 weeks, as indicated by decreased E-cadherin expression and an increased $\alpha$-smooth muscle actin ( $\alpha$-SMA). Allopurinol significantly inhibited UA-induced changes in E-cadherin and $\alpha$-SMA with an amelioration of kidney fibrosis at 6 weeks. In cultured rat renal tubular epithelial cells (NRK cells), UA induced EMT, which was blocked by the organic acid transport inhibitor, probenecid. UA increased expression of transcriptional factors associated with decreased synthesis of E-cadherin. UA also increased the degradation of E-cadherin via ubiquitination, which is of importance since downregulation of E-cadherin is considered to be a triggering mechanism for EMT. This study suggested that UA-induced EMT of renal tubular cells might be a novel mechanism explaining the association of hyperuricemia and renal progression after taking into account that EMT is an early phenomenon in kidney fibrosis [69-71]. Further research is required to assess the role of $\mathrm{UA}$ in other mesenchymal cell generation pathways.

\section{Perspective}

The underlying mechanisms by which UA could cause kidney fibrosis have been reported in animal models since 2001, and there has been a growing interest in this topic and numerous retrospective and prospective observational studies have been performed to assess the relationship between UA and CKD. The majority of data support the role of UA as a cause or exacerbating factor for kidney fibrosis and progressive $\mathrm{CKD}$. Although, over the last several years, some clinical intervention trials including randomized controlled trials (RCTs) have further supported this mechanistic role of UA [72-78], clinical evidence demonstrating the beneficial effect of UA lowering therapy with $\mathrm{XO}$ inhibitors on renoprotection and prevention of CKD progression is not certain yet and cannot be easily generalized. Most of the clinical studies are limited by their retrospective study design, small sample size, short follow-up duration, or lack of randomization, and no adequately powered RCT has yet demonstrated the beneficial effect of UA-lowering therapy on renal and cardiovascular outcomes in CKD [5]. Currently, there is no published clinical trial in which febuxostat was used as a XO inhibitor for evaluating the efficacy of UA reduction on progression of CKD.

With regard to the animal models in this research field, the uricase inhibitor OA-induced hyperuricemic rat model [26-29] was mainly used during the initial several years and all of the studies used allopurinol as a XO inhibitor. Thereafter, the research was performed using animal models of various kidney disease conditions, such as CsA nephropathy [36], diabetic nephropathy [38,51], obstructive nephropathy [61], ischemia-reperfusion injury [62], and 5/6 nephrectomy rat model [34]. Further research using diverse animal models of CKD $[79,80]$ is essential not only for gaining a better understanding of molecular mechanisms of kidney fibrosis but also for designing more appropriate clinical studies.

Febuxostat, a chemically engineered nonpurine selective inhibitor of XO, received approval in February 2009 from the Food and Drug Administration for the chronic management of hyperuricemia in patients with gout [81]. Although its clinical use is increasing, febuxostat is still generally considered as a second-line option for patients with gout who are unable to take allopurinol due to hypersensitivity, intolerance, renal insufficiency, or lack of efficacy in achieving a target serum UA level of $<6.0 \mathrm{mg} / \mathrm{dL}$. However, recently in basic research, febuxostat has largely replaced allopurinol as a XO inhibitor, and several animal studies have shown various beneficial effects of febuxostat in reducing inflammation, oxidative stress, and kidney fibrosis [34, 61, 62]. Febuxostat prevented renal injury in 5/6 nephrectomy rats with and without coexisting hyperuricemia [34]. But still there is little clinical experience and there are no clinical trials with febuxostat 


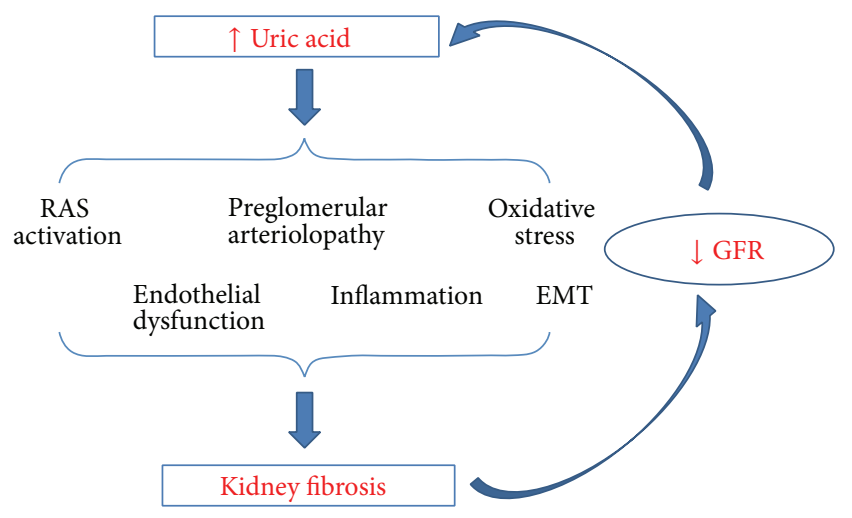

FIGURE 2: Mechanisms by which uric acid may cause kidney fibrosis based on experimental animal studies. EMT: epithelialmesenchymal transition; RAS: renin-angiotensin system.

assessing the efficacy of lowering UA on CKD progression, and it is also more expensive than allopurinol.

At present, drug therapy for asymptomatic hyperuricemia is not actively recommended, and this negative approach is mainly due to the absence of evidence from adequately powered RCTs on the causality between hyperuricemia and the onset or progression of CKD. Considering the large sample size required for an adequately powered trial, an international collaboration is necessary.

Current research interests focus on developing new effective antifibrotic drugs to slow progression or even reverse chronic kidney injury, and several compounds which target various components of the fibrotic pathway, from signaling molecules that include TGF- $\beta$, phosphatidylinositide3-kinase, and chemokines to microRNAs, are undergoing clinical trials $[82,83]$. Although, at present, UA may be one of the ignored risk factors for CKD and the clinical use of UA-lowering drugs which include allopurinol and febuxostat is largely confined to gout management, discovering the potential value of XO inhibitors for preventing kidney fibrosis and CKD progression will provide additional valuable tools for managing CKD.

\section{Conclusion}

After taking into account the results of all the important experimental studies mentioned above, UA and XO may contribute to kidney fibrosis mainly by inducing inflammation, endothelial dysfunction, oxidative stress, and activation of RAS (Figure 2). Besides, hyperuricemia induces alterations of renal hemodynamics via afferent arteriolopathy and contributes to the onset and progression of kidney fibrosis. Many experimental studies have shown that $\mathrm{XO}$ inhibitors may prevent kidney damage by lowering UA and inhibiting XO. However, there is no sufficient evidence from interventional clinical researches supporting the causal relationship between UA and kidney fibrosis, a final common pathway of CKD progression. Thus, the time is not quite appropriate for recommending the widespread clinical use of XO inhibitors for preventing CKD progression in patients with hyperuricemia and more so in patients without hyperuricemia. The effect and role of XO inhibitors in preventing kidney fibrosis and CKD progression must be further explored by performing future large scale clinical trials.

\section{Conflict of Interests}

The authors declare that there is no conflict of interests regarding the publication of this paper.

\section{References}

[1] S. B. Lee and R. Kalluri, "Mechanistic connection between inflammation and fibrosis," Kidney International, vol. 78, no. 119, pp. S22-S26, 2010.

[2] L. H. Beck, "Requiem for gouty nephropathy," Kidney International, vol. 30, no. 2, pp. 280-287, 1986.

[3] V. Filiopoulos, D. Hadjiyannakos, and D. Vlassopoulos, "New insights into uric acid effects on the progression and prognosis of chronic kidney disease," Renal Failure, vol. 34, no. 4, pp. 510520, 2012.

[4] D.-H. Kang and W. Chen, "Uric acid and chronic kidney disease: new understanding of an old problem," Seminars in Nephrology, vol. 31, no. 5, pp. 447-452, 2011.

[5] S. V. Badve, F. Brown, C. M. Hawley et al., "Challenges of conducting a trial of uric-acid-lowering therapy in CKD," Nature Reviews Nephrology, vol. 7, no. 5, pp. 295-300, 2011.

[6] D. I. Jalal, M. Chonchol, W. Chen, and G. Targher, "Uric acid as a target of therapy in CKD," The American Journal of Kidney Diseases, vol. 61, no. 1, pp. 134-146, 2013.

[7] R. J. Johnson, T. Nakagawa, D. Jalal, L. G. Sánchez-Lozada, D. H. Kang, and E. Ritz, "Uric acid and chronic kidney disease: which is chasing which?" Nephrology Dialysis Transplantation, vol. 28, no. 9, pp. 2221-2228, 2013.

[8] G. Bellomo, "Uric acid and chronic kidney disease: a time to act?" World Journal of Nephrology, vol. 2, no. 2, pp. 17-25, 2013.

[9] D. Gustafsson and R. Unwin, "The pathophysiology of hyperuricaemia and its possible relationship to cardiovascular disease, morbidity and mortality," BMC Nephrology, vol. 14, article 164, 2013.

[10] C. E. Berry and J. M. Hare, "Xanthine oxidoreductase and cardiovascular disease: molecular mechanisms and pathophysiological implications," Journal of Physiology, vol. 555, part 3, pp. 589-606, 2004.

[11] H. K. Choi, D. B. Mount, and A. M. Reginato, "Pathogenesis of gout," Annals of Internal Medicine, vol. 143, no. 7, pp. 499-516, 2005.

[12] S. Watanabe, D.-H. Kang, L. Feng et al., "Uric acid, hominoid evolution, and the pathogenesis of salt-sensitivity," Hypertension, vol. 40, no. 3, pp. 355-360, 2002.

[13] N. L. Edwards, "The role of hyperuricemia and gout in kidney and cardiovascular disease," Cleveland Clinic Journal of Medicine, vol. 75, supplement 5, pp. S13-S16, 2008.

[14] D. J. Levinson and L. B. Sorensen, "Renal handling of uric acid in normal and gouty subjects: evidence for a 4-component system," Annals of the Rheumatic Diseases, vol. 39, no. 2, pp. 173179, 1980.

[15] A. Taniguchi and N. Kamatani, "Control of renal uric acid excretion and gout," Current Opinion in Rheumatology, vol. 20, no. 2, pp. 192-197, 2008. 
[16] Y. Takano, K. Hase-Aoki, H. Horiuchi et al., "Selectivity of febuxostat, a novel non-purine inhibitor of xanthine oxidase/xanthine dehydrogenase," Life Sciences, vol. 76, no. 16, pp. 1835-1847, 2005.

[17] T. Hosoya, K. Kimura, S. Itoh et al., "The effect of febuxostat to prevent a further reduction in renal function of patients with hyperuricemia who have never had gout and are complicated by chronic kidney disease stage 3: study protocol for a multicenter randomized controlled study," Trials, vol. 15, no. 1, p. 26, 2014.

[18] B. A. Grabowski, R. Khosravan, L. Vernillet, and D. J. Mulford, "Metabolism and excretion of [14C] febuxostat, a novel nonpurine selective inhibitor of xanthine oxidase, in healthy male subjects," Journal of Clinical Pharmacology, vol. 51, no. 2, pp. 189-201, 2011.

[19] I. Garcia-Valladares, T. Khan, and L. R. Espinoza, "Efficacy and safety of febuxostat in patients with hyperuricemia and gout," Therapeutic Advances in Musculoskeletal Disease, vol. 3, no. 5, pp. 245-253, 2011.

[20] K. Komoriya, S. Hoshide, K. Takeda et al., "Pharmacokinetics and pharmacodynamics of febuxostat (TMX-67), a non-purine selective inhibitor of xanthine oxidase/xanthine dehydrogenase (NPSIXO) in patients with gout and/or hyperuricemia," Nucleosides, Nucleotides and Nucleic Acids, vol. 23, no. 8-9, pp. 1119$1122,2004$.

[21] Y. Osada, M. Tsuchimoto, H. Fukushima et al., "Hypouricemic effect of the novel xanthine oxidase inhibitor, TEI-6720, in rodents," European Journal of Pharmacology, vol. 241, no. 2-3, pp. 183-188, 1993.

[22] S. P. Bruce, "Febuxostat: a selective xanthine oxidase inhibitor for the treatment of hyperuricemia and gout," Annals of Pharmacotherapy, vol. 40, no. 12, pp. 2187-2194, 2006.

[23] C. L. Gray and N. E. Walters-Smith, "Febuxostat for treatment of chronic gout," The American Journal of Health-System Pharmacy, vol. 68, no. 5, pp. 389-398, 2011.

[24] R. J. Johnson, S. D. Kivlighn, Y.-G. Kim, S. Suga, and A. B. Fogo, "Reappraisal of the pathogenesis and consequences of hyperuricemia in hypertension, cardiovascular disease, and renal disease," The American Journal of Kidney Diseases, vol. 33, no. 2, pp. 225-234, 1999.

[25] X. Wu, M. Wakamiya, S. Vaishnav et al., "Hyperuricemia and urate nephropathy in urate oxidase-deficient mice," Proceedings of the National Academy of Sciences of the United States of America, vol. 91, no. 2, pp. 742-746, 1994.

[26] M. Mazzali, J. Hughes, Y.-G. Kim et al., "Elevated uric acid increases blood pressure in the rat by a novel crystalindependent mechanism," Hypertension, vol. 38, no. 5, pp. 11011106, 2001.

[27] M. Mazzali, J. Kanellis, L. Han et al., "Hyperuricemia induces a primary renal arteriolopathy in rats by a blood pressure-independent mechanism," The American Journal of Physiology_Renal Physiology, vol. 282, no. 6, pp. F991-F997, 2002.

[28] L. G. Sánchez-Lozada, E. Tapia, J. Santamaría et al., "Mild hyperuricemia induces vasoconstriction and maintains glomerular hypertension in normal and remnant kidney rats," Kidney International, vol. 67, no. 1, pp. 237-247, 2005.

[29] D.-H. Kang, T. Nakagawa, L. Feng et al., "A role for uric acid in the progression of renal disease," Journal of the American Society of Nephrology, vol. 13, no. 12, pp. 2888-2897, 2002.

[30] V. Diwan, A. Mistry, G. Gobe, and L. Brown, "Adenine-induced chronic kidney and cardiovascular damage in rats," Journal of
Pharmacological and Toxicological Methods, vol. 68, no. 2, pp. 197-207, 2013.

[31] M. Correa-Costa, T. T. Braga, P. Semedo et al., "Pivotal role of toll-like receptors 2 and 4, its adaptor molecule MyD88, and inflammasome complex in experimental tubule-interstitial nephritis," PLoS ONE, vol. 6, no. 12, Article ID e29004, 2011.

[32] L. G. Sánchez-Lozada, E. Tapia, V. Soto et al., "Treatment with the xanthine oxidase inhibitor febuxostat lowers uric acid and alleviates systemic and glomerular hypertension in experimental hyperuricaemia," Nephrology Dialysis Transplantation, vol. 23, no. 4, pp. 1179-1185, 2008.

[33] L. G. Sánchez-Lozada, E. Tapia, C. Avila-Casado et al., "Mild hyperuricemia induces glomerular hypertension in normal rats," The American Journal of Physiology - Renal Physiology, vol. 283, no. 5, pp. F1105-F1110, 2002.

[34] L. G. Sánchez-Lozada, E. Tapia, V. Soto et al., "Effect of febuxostat on the progression of renal disease in 5/6 nephrectomy rats with and without hyperuricemia," Nephron-Physiology, vol. 108, no. 4, pp. p69-p78, 2008.

[35] M. Mazzali, Y.-G. Kim, S.-I. Suga et al., "Hyperuricemia exacerbates chronic cyclosporine nephropathy," Transplantation, vol. 71, no. 7, pp. 900-905, 2001.

[36] F. C. Mazali, R. J. Johnson, and M. Mazzali, "Use of uric acidlowering agents limits experimental cyclosporine nephropathy," Nephron-Experimental Nephrology, vol. 120, no. 1, pp. el2-e19, 2012.

[37] T. Kosugi, T. Nakayama, M. Heinig et al., "Effect of lowering uric acid on renal disease in the type 2 diabetic $\mathrm{db} / \mathrm{db}$ mice," The American Journal of Physiology - Renal Physiology, vol. 297, no. 2, pp. F481-F488, 2009.

[38] S. M. Kim, Y. W. Choi, H. Y. Seok et al., "Reducing serum uric acid attenuates TGF- $\beta 1$-induced profibrogenic progression in type 2 diabetic nephropathy," Nephron Experimental Nephrology, vol. 121, no. 3-4, pp. e109-e121, 2012.

[39] B. N. Ames, R. Cathcart, E. Schwiers, and P. Hochstein, "Uric acid provides an antioxidant defense in humans against oxidant- and radical-caused aging and cancer: a hypothesis," Proceedings of the National Academy of Sciences of the United States of America, vol. 78, no. 11, pp. 6858-6862, 1981.

[40] D. B. Corry, P. Eslami, K. Yamamoto, M. D. Nyby, H. Makino, and M. L. Tuck, "Uric acid stimulates vascular smooth muscle cell proliferation and oxidative stress via the vascular reninangiotensin system," Journal of Hypertension, vol. 26, no. 2, pp. 269-275, 2008.

[41] Y. Y. Sautin, T. Nakagawa, S. Zharikov, and R. J. Johnson, "Adverse effects of the classic antioxidant uric acid in adipocytes: NADPH oxidase-mediated oxidative/nitrosative stress," The American Journal of Physiology-Cell Physiology, vol. 293, no. 2, pp. C584-C596, 2007.

[42] Y. Y. Sautin and R. J. Johnson, "Uric acid: the oxidantantioxidant paradox," Nucleosides, Nucleotides and Nucleic Acids, vol. 27, no. 6-7, pp. 608-619, 2008.

[43] B. Frei, R. Stocker, and B. N. Ames, "Antioxidant defenses and lipid peroxidation in human blood plasma," Proceedings of the National Academy of Sciences of the United States of America, vol. 85, no. 24, pp. 9748-9752, 1988.

[44] Z. F. Yu, A. J. Bruce-Keller, Y. Goodman, and M. P. Mattson, "Uric acid protects neurons against excitotoxic and metabolic insults in cell culture, and against focal ischemic brain injury in vivo," Journal of Neuroscience Research, vol. 53, no. 5, pp. 613625, 1998. 
[45] D. C. Hooper, S. Spitsin, R. B. Kean et al., "Uric acid, a natural scavenger of peroxynitrite, in experimental allergic encephalomyelitis and multiple sclerosis," Proceedings of the National Academy of Sciences of the United States of America, vol. 95, no. 2, pp. 675-680, 1998.

[46] K. Tsukada, T. Hasegawa, S. Tsutsumi et al., "Effect of uric acid on liver injury during hemorrhagic shock," Surgery, vol. 127, no. 4, pp. 439-446, 2000.

[47] B. F. Becker, N. Reinholz, T. Ozcelik, B. Leipert, and E. Gerlach, "Uric acid as radical scavenger and antioxidant in the heart," Pflugers Archiv European Journal of Physiology, vol. 415, no. 2, pp. 127-135, 1989.

[48] L. G. Sánchez-Lozada, V. Soto, E. Tapia et al., "Role of oxidative stress in the renal abnormalities induced by experimental hyperuricemia," The American Journal of Physiology-Renal Physiology, vol. 295, no. 4, pp. F1134-F1141, 2008.

[49] L. G. Sánchez-Lozada, E. Tapia, R. López-Molina et al., "Effects of acute and chronic L-arginine treatment in experimental hyperuricemia," The American Journal of Physiology-Renal Physiology, vol. 292, no. 4, pp. 1238-1244, 2007.

[50] L. G. Sánchez-Lozada, M. A. Lanaspa, M. Cristóbal-García et al., "Uric acid-induced endothelial dysfunction is associated with mitochondrial alterations and decreased intracellular ATP concentrations," Nephron Experimental Nephrology, vol. 121, no. 3-4, pp. e71-e78, 2012.

[51] S. J. Hwang, K. H. Lee, H. H. Jang et al., "Febuxostat contributes to improvement of endothelial dysfunction in an experimental model of streptozocin-induced diabetic rats," International Journal of Cardiology, vol. 171, no. 3, pp. el10-e112, 2014.

[52] J. Kanellis, S. Watanabe, J. H. Li et al., "Uric acid stimulates monocyte chemoattractant protein-1 production in vascular smooth muscle cells via mitogen-activated protein kinase and cyclooxygenase-2," Hypertension, vol. 41, no. 6, pp. 1287-1293, 2003.

[53] D.-H. Kang, S.-K. Park, I.-K. Lee, and R. J. Johnson, "Uric acid-induced C-reactive protein expression: implication on cell proliferation and nitric oxide production of human vascular cells," Journal of the American Society of Nephrology, vol. 16, no. 12, pp. 3553-3562, 2005.

[54] H. J. Han, M. J. Lim, Y. J. Lee, J. H. Lee, I. S. Yang, and M. Taub, "Uric acid inhibits renal proximal tubule cell proliferation via at least two signaling pathways involving PKC, MAPK, cPLA2, and NF- $\kappa \mathrm{B}$," The American Journal of Physiology-Renal Physiology, vol. 292, no. 1, pp. F373-F381, 2007.

[55] Z. Yang, W. Xiaohua, J. Lei et al., "Uric acid increases fibronectin synthesis through upregulation of lysyl oxidase expression in rat renal tubular epithelial cells," The American Journal of Physiology-Renal Physiology, vol. 299, no. 2, pp. F336-F346, 2010.

[56] J. Nomura, N. Busso, A. Ives et al., "Febuxostat, an inhibitor of xanthine oxidase, suppresses lipopolysaccharide-induced MCP-1 production via MAPK phosphatase-1-mediated inactivation of JNK," PLoS ONE, vol. 8, no. 9, Article ID e75527, 2013.

[57] R. P. Brandes, G. Koddenberg, W. Gwinner et al., "Role of increased production of superoxide anions by $\mathrm{NAD}(\mathrm{P}) \mathrm{H}$ oxidase and xanthine oxidase in prolonged endotoxemia," Hypertension, vol. 33, no. 5, pp. 1243-1249, 1999.

[58] P. M. Hassoun, F.-S. Yu, C. G. Cote et al., "Upregulation of xanthine oxidase by lipopolysaccharide, interleukin-1, and hypoxia: role in acute lung injury," The American Journal of Respiratory and Critical Care Medicine, vol. 158, no. 1, pp. 299305, 1998.
[59] U. S. Kayyali, C. Donaldson, H. Huang, R. Abdelnour, and P. M. Hassoun, "Phosphorylation of xanthine dehydrogenase/oxidase in hypoxia," Journal of Biological Chemistry, vol. 276, no. 17, pp. 14359-14365, 2001.

[60] K. Nakai, M. B. Kadiiska, J.-J. Jiang, K. Stadler, and R. P. Mason, "Free radical production requires both inducible nitric oxide synthase and xanthine oxidase in LPS-treated skin," Proceedings of the National Academy of Sciences of the United States of America, vol. 103, no. 12, pp. 4616-4621, 2006.

[61] H. Omori, N. Kawada, K. Inoue et al., "Use of xanthine oxidase inhibitor febuxostat inhibits renal interstitial inflammation and fibrosis in unilateral ureteral obstructive nephropathy," Clinical and Experimental Nephrology, vol. 16, pp. 549-556, 2012.

[62] H. Tsuda, N. Kawada, J. Y. Kaimori et al., "Febuxostat suppressed renal ischemia-reperfusion injury via reduced oxidative stress," Biochemical and Biophysical Research Communications, vol. 427, no. 2, pp. 266-272, 2012.

[63] A. Kushiyama, H. Okubo, H. Sakoda et al., "Xanthine oxidoreductase is involved in macrophage foam cell formation and atherosclerosis development," Arteriosclerosis, Thrombosis, and Vascular Biology, vol. 32, no. 2, pp. 291-298, 2012.

[64] K. Schröder, C. Vecchione, O. Jung et al., "Xanthine oxidase inhibitor tungsten prevents the development of atherosclerosis in ApoE knockout mice fed a Western-type diet," Free Radical Biology and Medicine, vol. 41, no. 9, pp. 1353-1360, 2006.

[65] N. Engberding, S. Spiekermann, A. Schaefer et al., "Allopurinol attenuates left ventricular remodeling and dysfunction after experimental myocardial infarction: a new action for an old drug?" Circulation, vol. 110, no. 15, pp. 2175-2179, 2004.

[66] R. M. Wright, L. A. Ginger, N. Kosila et al., "Mononuclear phagocyte xanthine oxidoreductase contributes to cytokineinduced acute lung injury," The American Journal of Respiratory Cell and Molecular Biology, vol. 30, no. 4, pp. 479-490, 2004.

[67] Y. Liu, "New insights into epithelial-mesenchymal transition in kidney fibrosis," Journal of the American Society of Nephrology, vol. 21, no. 2, pp. 212-222, 2010.

[68] R. M. Carew, B. Wang, and P. Kantharidis, "The role of EMT in renal fibrosis," Cell and Tissue Research, vol. 347, no. 1, pp. 103116, 2012.

[69] E. S. Ryu, M. J. Kim, H. S. Shin et al., "Uric acid-induced phenotypic transition of renal tubular cells as a novel mechanism of chronic kidney disease," The American Journal of PhysiologyRenal Physiology, vol. 304, no. 5, pp. F471-F480, 2013.

[70] R. Kalluri, "EMT: when epithelial cells decide to become mesenchymal-like cells," Journal of Clinical Investigation, vol. 119, no. 6, pp. 1417-1419, 2009.

[71] R. Kalluri and E. G. Neilson, "Epithelial-mesenchymal transition and its implications for fibrosis," Journal of Clinical Investigation, vol. 112, no. 12, pp. 1776-1784, 2003.

[72] D. A. J. Neal, B. D. M. Tom, A. E. S. Gimson, P. Gibbs, and G. J. M. Alexander, "Hyperuricemia, gout, and renal function after liver transplantation," Transplantation, vol. 72, no. 10, pp. 16891691, 2001.

[73] L. D. Fairbanks, J. S. Cameron, G. Venkat-Raman et al., "Early treatment with allopurinol in familial juvenil hyerpuricaemic nephropathy (FJHN) ameliorates the long-term progression of renal disease," QJM-Monthly Journal of the Association of Physicians, vol. 95, no. 9, pp. 597-607, 2002.

[74] Y.-P. Siu, K.-T. Leung, M. K.-H. Tong, and T.-H. Kwan, "Use of allopurinol in slowing the progression of renal disease through its ability to lower serum uric acid level," The American Journal of Kidney Diseases, vol. 47, no. 1, pp. 51-59, 2006. 
[75] M. Kanbay, A. Ozkara, Y. Selcoki et al., "Effect of treatment of hyperuricemia with allopurinol on blood pressure, creatinine clearence, and proteinuria in patients with normal renal functions," International Urology and Nephrology, vol. 39, no. 4, pp. 1227-1233, 2007.

[76] M. Kanbay, B. Afsar, and A. Covic, "Uric acid as a cardiometabolic risk factor: to be or not to be," Contributions to Nephrology, vol. 171, pp. 62-67, 2011.

[77] M. Goicoechea, S. G. de Vinuesa, U. Verdalles et al., "Effect of allopurinol in chronic kidney disease progression and cardiovascular risk," Clinical Journal of the American Society of Nephrology, vol. 5, no. 8, pp. 1388-1393, 2010.

[78] B. H. Pai, G. Swarnalatha, R. Ram, and K. V. Dakshinamurty, "Allopurinol for prevention of progression of kidney disease with hyperuricemia," Indian Journal of Nephrology, vol. 23, no. 4, pp. 280-286, 2013.

[79] H.-C. Yang, Y. Zuo, and A. B. Fogo, "Models of chronic kidney disease," Drug Discovery Today: Disease Models, vol. 7, no. 1-2, pp. 13-19, 2010.

[80] G. J. Becker and T. D. Hewitson, "Animal models of chronic kidney disease: useful but not perfect," Nephrology Dialysis Transplantation, vol. 28, no. 10, pp. 2432-2438, 2013.

[81] "Febuxostat (Uloric) for chronic treatment of gout," The Medical letter on drugs and therapeutics, vol. 51, no. 1312, pp. 37-38, 2009.

[82] D. Tampe and M. Zeisberg, "Potential approaches to reverse or repair renal fibrosis," Nature Reviews Nephrology, vol. 10, no. 4, pp. 226-237, 2014.

[83] S. P. Srivastava, D. Koya, and K. Kanasaki, "MicroRNAs in kidney fibrosis and diabetic nephropathy: roles on EMT and EndMT,' BioMed Research International, vol. 2013, Article ID 125469, 10 pages, 2013. 


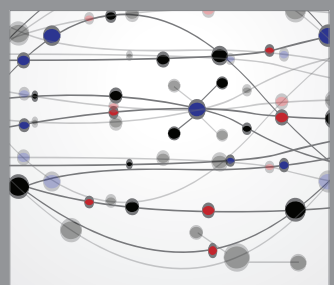

The Scientific World Journal
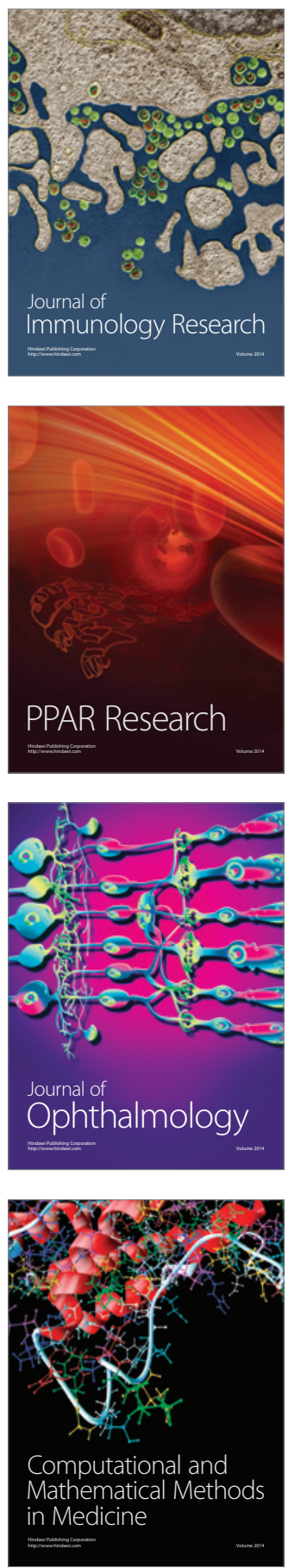

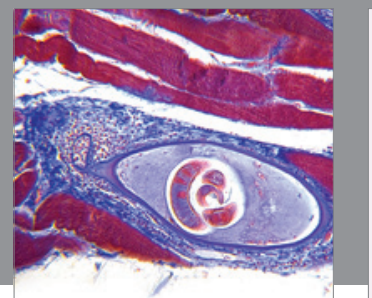

Gastroenterology

Research and Practice
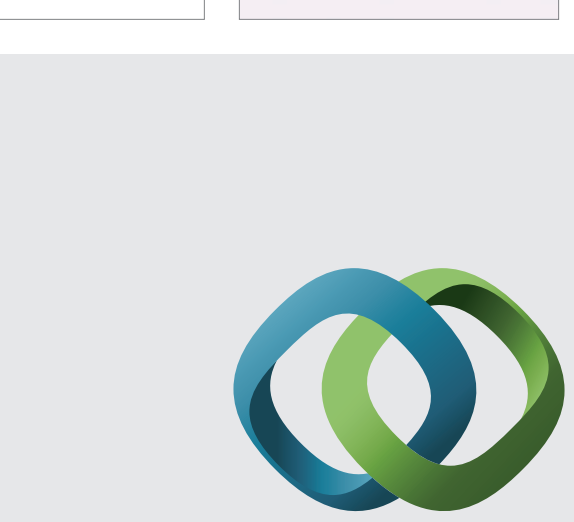

\section{Hindawi}

Submit your manuscripts at

http://www.hindawi.com
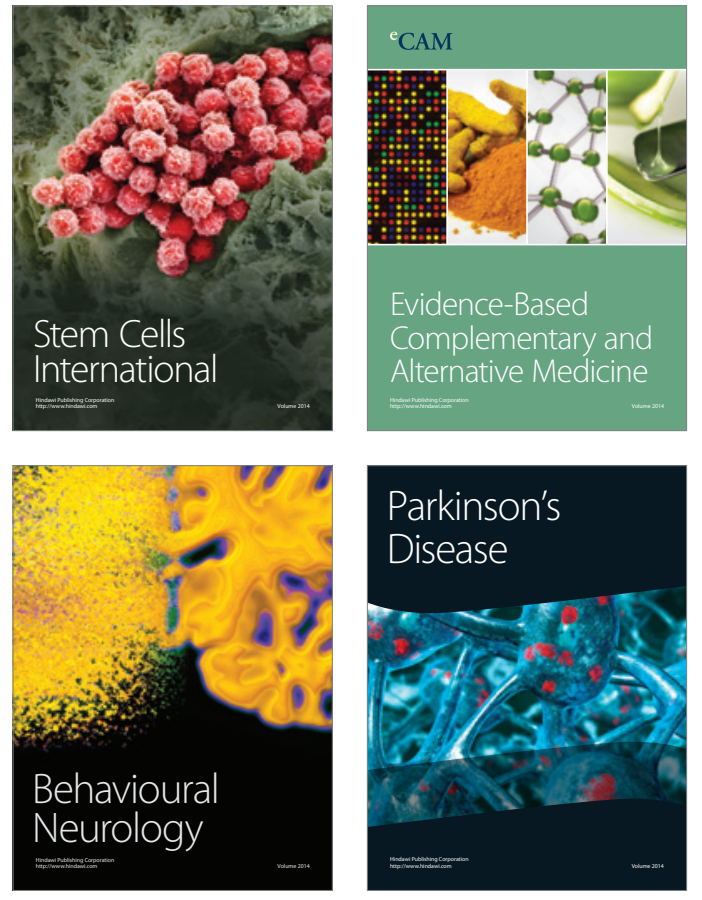
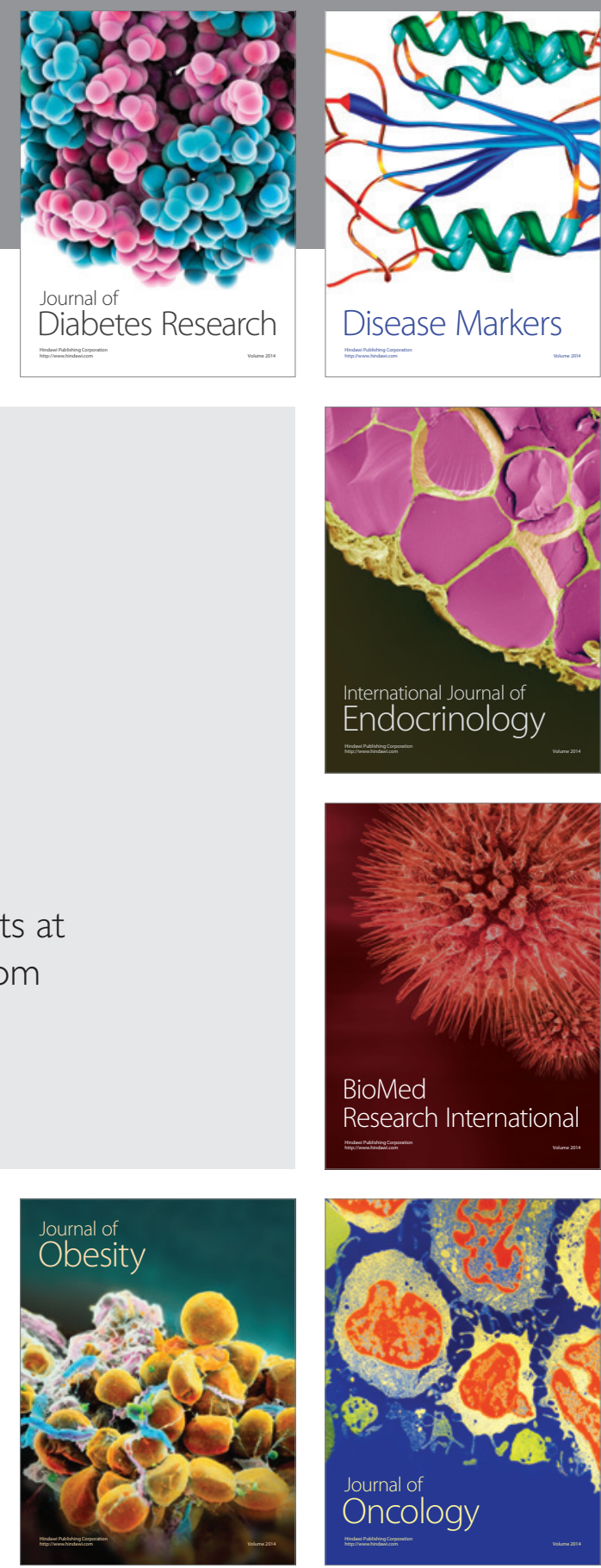

Disease Markers
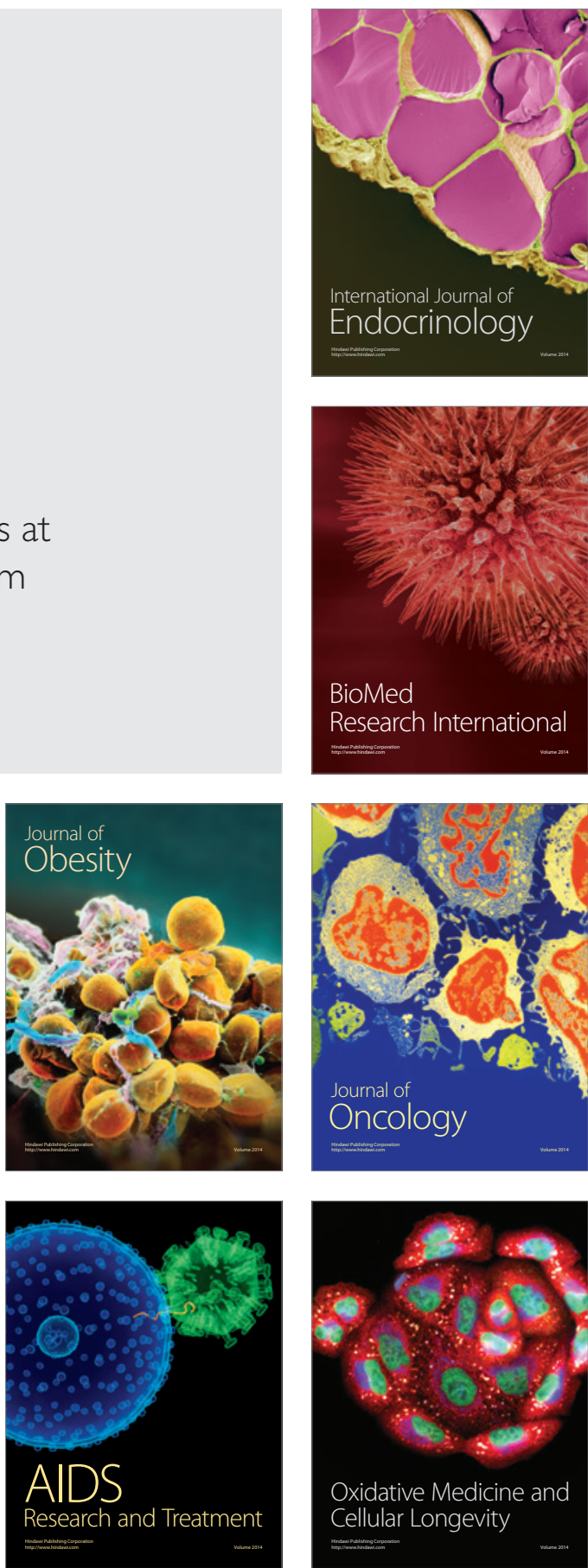